\title{
Editorial
}

\section{Reduction of Medical Errors: An Order}

\section{Gilbert I. Martin, MD}

An article appeared on the front page of the December 12, 1999 issue of the New York Times entitled: "A Clinton Order Seeks to Reduce Medical Errors." The material commented on an earlier report by the National Academy of Sciences which said that medical mistakes were responsible for tens of thousands of deaths each year. The suggestion was made that health care providers collect and analyze information on unsafe practices and attempt to affect change. Thus far, there are some providers and states that investigate these errors, but compliance and organization are at best "spotty." President Clinton ordered federal agencies to take steps to reduce these medical mistakes. Standards would be developed that would be under federal control; because the government subsidizes the majority of health care in the United States, enforcement would have financial implications.

Quality assurance has been a "buzz phrase" in the last few years, and these collective quality improvement programs have spawned a new cottage industry. The concept of iatrogenic disease is certainly not new. The word "iatrogenic" is derived from two Greek words and means "physician induced." History tells us that as medicine progressed, psychiatry used the term to infer that were misinterpretations of physicians' attitudes and comments toward their patients. Today, the term iatrogenic to many of us means the inadvertent side effects and complications that may be created over the course of diagnosis and treatment. I believe that in the future, the term will become one that is a disease of "medical progress." We have neonatologists, perinatologists, and gerontologists, and soon we will have iatrogentolo- gists who will specialize in the prevention of these "inadvertent effects and complications."

In truth, the National Academy of Sciences report was sobering. In the United States, 3\% to 5\% of all hospital admissions are secondary to drug reactions. Hospital-acquired infections and postoperative wound infections constitute another group of patients, and many hospital deaths are associated with a therapeutic or diagnostic procedure. Medication errors are especially noticeable, as illegible physician orders and incorrect drug dosages or drug interactions may lead to disastrous consequences. Nursing has attempted to decrease the number mistakes by double-checking computations and reporting medication incidents. The involvement of the "pharmacist" is of additional benefit.

Perinatologists and Neonatologists can take a leading role in augmenting the quality assurance process to decrease mortality and morbidity. As hospitalists, we are intimately involved with all aspects of inpatient care. There are individual programs available to assist the physician with orders (which will be legible), dose amounts, and schedules. We can identify mortality and morbidity and collect and analyze data. Once a specific problem has been identified, recommendations can be presented that over time will lead to discussion and ideally elimination of the error. Our subspecialties have been involved in collecting information to improve perinatal care, and the mechanisms are already in place.

It would seem natural that we take the lead in demonstrating to the National Academy of Sciences and the federal government that medical errors can in fact be substantially reduced and consequently a decrease in mortality and morbidity can be achieved. Of course, this means that the subspecialty of "iatrogentologist" may disappear. 